andere Metalloxyde vorhanden sind, welche durch Ferridcyankalium ebenfalls gefällt werden.

Zur. Ausführung der Reaktion bringt man einen kleinen Teil der auf Eisenoxydul zu prüfenden Lösung in einen Reagenszylinder; gibt das gleiche Volumen konzentrierte Schwefelsäure hinzu, kühlt ab und lässt bei schief gehaltenem Zylinder einen grösseren Kristall Kaliumnitrat in die so präparierte Lösung eingleiten. Hierauf bewegt man den Zylinder ein wenig und beobachtet die Färbung. Durch das Auftreten der oben erwähnten Rot- bis Braunfärbung ist die Gegenwart des Eisenoxyduls erwiesen.

Sind grössere Mengen von Chlormetallen vorhanden, welche die Empfindlichkeit der Reaktion beeinträchtigen können, so erhitzt man nach dem Zusatz von Schwefelsäure bis zum Sieden und verfährt dann erst, wie angegeben. Hierdurch werden die Chloride in Sulfate übergerührt, und gleichzeitig wird die in Freiheit gesetzte Chlorwasserstoffsäure durch das Kochen grösstenteils ausgetrieben.

Esch a. d. Alzette, November 1904.

Qualitativer Nachweis des Zinns in seinen 0xydulverbindungen. Von

\title{
I. Blum.
}

Die in vorstehender Mitteilung beschriebene Reaktion zum Nachweis des Eisenoxyduls kann auch auf indirektem Wege zum Nachweis des Zinns in seinen Oxydulverbindungen dienen.

Versetzt man eine Zinnoxydul oder Zinnchlorür und Salzsäure enthaltende Lösung mit einem Tropfen Eisenchlorid, so wird eine dem vorhandenen Stannogehalte entsprechende Menge Eisenchlorid zu Eisenchlorür reduziert. Versetzt man nun weiter die Lösung mit konzentrierter Schwefelsäure, kühlt ab und gibt einen Kristall Kaliumnitrat hinzu, so lässt sich an der auftretenden Rot- bis Braunfärbung das entstandene Eisenchlorür und hierdurch indirekt das vorhandene Zinnchlorür nachweisen. Andere Eisenchlorid reduzierende Substanzen dürfen nicht zugegen sein.

Bringt man aus einem Gemenge, welches Schwefelarsen, Schwefelantimon und Schwefelzinn enthält, durch Erhitzen mit rauchender Salzsäure in gelinder Wärme die beiden letzteren in Lösung, erhitzt bis 
zum Verjagen des Schwefelwasserstoffs, verdünnt mit Wasser und filtriert, so kann man in einem Teilchen des Filtrates nach der angegebenen Reaktion auf Zinn prüfen. $\mathrm{Zu}$ diesem $Z$ wecke gibt man einen Teil der auf Zinn und Antimon zu untersuchenden Lösung in einen Reagenszylinder, versetzt mit einigen Tropfen Eisen hhloridlösung, gibt etwa das gleiche Volumen konzentrierte Schwefelsäure hinzu, kühlt ab und lässt einen grösseren Kristall Kaliumnitrat in die Lösung eingleiten. Man bewegt den Reagenszylinder ein wenig und beobachtet das Erscheinen der , Färbung. Tritt Rot- bis Braunfärbung ein, dann ist damit die Gegenwart des Zinns nachgewiesen.

Esch a. d. Alzette, November 1904.

\section{Über die alkalische Reaktion von Strontium- und Kalziumkarbonat. Von}

\section{Blum.}

Nach den in der chemischen Literatur vorhandenen Angaben ist die alkalische Reaktion von Baryum- und Magnesiumkarbonat bekannt'). Dasselbe ist nicht der Fall für Strontium- und Kalziumkarbonat. Hierauf bezügliche Angaben konnte ich in, der Literatur nicht finden.

$\mathrm{Zu}$ den folgenden Versuchen diente Strontiumkarbonat, welches durch Umsetzen von Strontiumnitrat und Kalziumkarbonat, vollständiges Auswaschen der Nieders'bläge und Trocknen derselben bei $120^{\circ}$ erhalten wurde. Das Kalziumkarbonat war dargestellt durch Einwirkung von Ammoniumkarbonat auf Chlorkalzium.

Zum Nachweis der alkalischen Reaktion der beiden Verbindungen wurde ein Streifen angefeuchtetes, rotes Lackmuspapier auf die konkave Seite eines Uhrglases angedrückt, eine Messerspitze voll der betreffenden Verbindung auf die Mitte des Papierstreifens aufgetrayen und mit einigen Tropfen Wasser versetzt. Es trat bei den beiden Verbindungen nach etwa 5 Minuten eine Blaufärbung des roten Lackmuspapierstreifens ein. welche sich auf die ganze Fläche erstreckte, die von dem Strontiumund Kalziumkarbonatbrei bedeckt wurde. Damit ist die alkalische Reaktion der beiden Karbonate erwiesen.

1) Vergleiche hierüber u. a, $\mathrm{H}$ a g er, Handbuch der pharmazentischen Praxis. 